\title{
Analysis and Design of Reinforced Concrete Multi-Stored Building (G+5) by using Tekla Software
}

\author{
P.Nagasri Anjaneyulu ${ }^{1}$ | Dr. Dumpa Venkateswarlu ${ }^{2}$ \\ ${ }^{1}$ PG Scholar, Department of Civil Engineering, Godavari Institute of Engineering \& Technology, Rajahmundry \\ ${ }^{2}$ Professor \& Head, Department of Civil Engineering, Godavari Institute of Engineering \& Technology, Rajahmundry
}

To Cite this Article

P.Nagasri Anjaneyulu and Dr. Dumpa Venkateswarlu, "Analysis and Design of Reinforced Concrete Multi-Stored Building $(\mathrm{G}+5)$ by using Tekla Software", International Journal for Modern Trends in Science and Technology, Vol. 07, Issue 03, March 2021, pp.: 229-244.

Article Info

Received on 15-February-2021, Revised on 12-March-2021, Accepted on 17-March-2021, Published on 20-March-2021.

\section{ABSTRACT}

The rapidly developing world, in construction industry software's are used today is the urgent necessity of the moment in order to match the peace of infrastructural development. The main objective of the study is checking the computability of results. The analysis and design of Reinforced Concrete structures is a final stage in the construction industry to deliver the projects with in the estimated time and within the budget. Analysis and design tasks is not an easy matter of fact. Especially specialized software's are used such as Robot structural Analysis, MIDAS Gen, SAP2000, STAAD PRO, ETABS, TEKLA Structural designer, S-Frame and so on. To gain the design results most commonly used software are validated. In manual design process Indian Standard Codes are utilized and different design modules are used to gain the confidence of the users. The basic elements of a RC Structure such as beams, columns and simple frame have been discussed. In analysis process, shear force and bending moment of beams and columns are comparable in all cases. Thereafter the loads are calculated namely the dead loads, which depend on the unit weight of the materials used (concrete, brick), live loads, which according to the code IS 875-1987 and earthquake load according to IS1893 (PART-1). IS 456:2000 code of practice for plain and reinforced concrete and IS 800:2007 limit state design and seismic ductile design IS:13920(2016) and seismic loading IS 1893 (part 1):2016 and wind loading IS875(part 3):1987. Finally, the footings are designed based on loading from the column and the soil bearing capacity for the particular area. Most importantly, the sections must be checked for all the four components with regard to strength and serviceability factors. Construction plays a significant role in the emergence of ecological problems such as environmental pollutions, energy consumption and carbon emission and so on. In the emergence of ecological situations the specific attention has to be devoted and develop the sustainable and green solutions in the design of buildings and structures.

KEYWORDS:Geometry, Structure, Analysis, Shear force, Bending moment, Deflection, Wind analysis, Seismic analysis, Finite element analysis, Torsion, Static and Dynamic analysis`s.

\section{INTRODUCTION}

Computer Literacy started increasing rapidly from 1994 and by 2000 there were thousands of companies offering software, website solution to various requirements. During that time Indian software professionals were busy with fixing the Y2K bug. During Dot com bust in 2000-01, many companies invested heavily on software's, 
hardware's and few companies took full advantage of computerization where as many other companies lost a lot of money by doing wrong investment.

By 2001-02 many software companies started selling half-baked software and companies who choose wrong software products started burning their fingers. Then a new trend started, where buyers stated investing wisely on proven solutions. Slowly Software development companies who did not have sufficient skill started closing their shutters. The software has now become like any other commodity where there are many options to choose from. Now generally it is easy to find a good solution for any requirement. As only stable products are surviving in the market and sufficient, product reviews are in place.

With Software products becoming stable selecting the right software for your requirements is not a difficult task, but a little bit of background check is certainly required and the people who use the software have to be involved in decision making.

According to Madam Tan, Tekla has numerous attributes that make it ideal for steel fabricators. For example, it is able to handle changes on the fly so any changes proposed by the architect to the original drawing are automatically reflected in the general arrangement drawing.

For an engineer who is new to designing multi-storey buildings it is important that they follow a logical sequence through the various stages of the design process. Six steps that define this sequence are described below. Rules of thumb are included within each step to help the designer quickly and efficiently arrive at a solution that is sensible for a given set of constraints. In addition, it is important for the designer to understand some overarching principles of good design - sothat the result is not only sensible but is also 'good'.

The stages of a construction project are presented in ISE publication 'structural design -the engineer's role. Which may be broadly summarized as follows:

- Project formulation - What it's for, why is it being proposed, where is it, etc.

- Assembling the data and developing the brief - Understanding the site and context.

- Scheme design - Looking at and developing options.

- Detailed drawing - Of the various components and elements.
- Information for construction - Drawings, specifications.

- Construction.

Many of these stages include aspects of engineering design. A characteristic of RCC framed construction is that the constituent parts of the structure are manufactured off-site, with all the quality and speed-on-site benefits that are associated with such a form of construction. An implication of this, however, is that the design must be substantially complete before construction (concrete work fabrication) can begin. It is therefore important that the designer follows a logical sequence, as going back and revisiting earlier design decisions, once other parties involved have moved on to designing other parts of the building or manufacturing components, can be disproportionately expensive.

\section{Objectives:}

To perform analysis and design of the structure without any type of failures.

- To understand the basic principles of the structures by using Indian standared codes.

- To understand the parameters of the design for beams, column, slabs and other structural components.

- To prepare the 3D models of the structure by using the Tekla software for detailed analysis and design.

\section{Scope:}

The intent of this report is to present a detailed description of the aspects of structural analysis and structural design of RC components in Multi-stored building, ensuring the requirements of functional utility and structural integrity of the structure. The scope of this design report covers the following aspects:

a) Material properties and foundation parameters considered.

b) Mathematical modelling related aspects.

c) Results of Static analysis.

d) Results of Seismic Analysis.

e) RC design of various structural components Beams, columns and slabs.

f) Foundation analysis and design.

This report is to be read in conjunction with the multi-storey building structure. 


\section{RELATED WORK}

\section{Mohit Sharma et. al. (2015). To study the dynamic analysis of multi-storey Building.}

He considered a $\mathrm{G}+30$ storied regular reinforced concrete framed building. Dynamic analysis of multi-storey Building was carried out. These buildings have the plan area of $25 \mathrm{~m} \times 45 \mathrm{~m}$ with a storey height $3.6 \mathrm{~m}$ each and depth of foundation is $2.4 \mathrm{~m}$. \& total height of chosen building including depth of foundation is $114 \mathrm{~m}$. The static and dynamic analysis has done on computer with the help of STAAD-Pro software using the parameters for the design as per the IS-1893-2002-Part-1 for the zones- 2 and 3. It was concluded that not much difference in the values of Axial Forces as obtained by static and dynamic analysis.

\section{Prakash Sangamnerkar et al. (2015). Static and dynamic behavior of reinforced concrete framed regular building.}

He has done the comparative study on the static and dynamic behavior of reinforced concrete framed regular building. Comparison of static and vibrant behavior of a six storey'sstructure is considered in this paper and it is analyzed by using computerized solution available in all four seismic zones i.e. II, III, IV and V. This is important for building design and resistant from earthquake.

Project name: Luminary

Project category: Commercial

Company: A-Insinoorit Suunnittelu Oy, Skanska Oy, BST-Arkkitehdit Oy, Betonirakenne Oinas Oy, Parma Oy, Optiplan Oy, Caverion Suomi Oy

Project location: Finland

A. A unique tall hybrid building:

Judging by its architecture and appearance, Luminary is a uniquely tall and high-class complex of apartments and business, office and parking space. The building encompasses 188 apartments from studios to large penthouses. The business section includes a restaurant and a cafe with a roof terrace.

On the second floor, the large lobby functions as a hub of different areas, which represents exceptional architecture in Finland. The tower section houses 21 floors. Once completed, it will be the tallest apartment house in the Pirkanmaa region $(70.52 \mathrm{~m})$ and the new landmark of Tampere. Building regulations for tall buildings have affected the facade materials and exits. The building comprises $18,000 \mathrm{brm}^{2}$ in total.

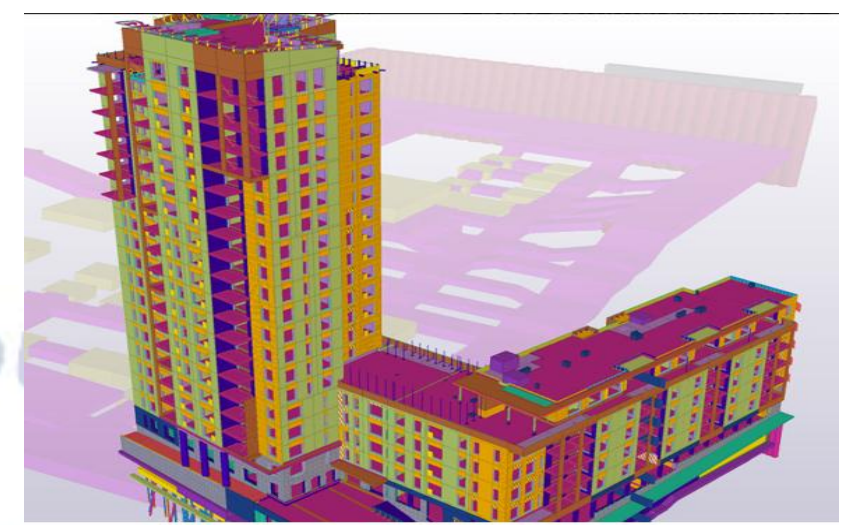

Regarding technology, engineering and trims, the apartments feature exceptional quality. Large floor-to-ceiling windows, a shared roof terrace and new type of services, such as car sharing, add luxury to the life of the residents. The yard is built on top of a two-floor parking garage. Luminary is located by a future tram route and very close to the main railway station.

Facade maintenance has been designed carefully using the model. There is a maintenance cradle on the roof that can circle the building on a monorail, and its reach for window cleaning and facade trimming has been ensured using the model. The building mainly consists of precast concrete units, but the lowest floors are cast in place. The roof structures, monorails and exit stairs are all made of steel. Construction started in August 2016, and the project is to be completed in March 2019.

Model data frequently utilized on the construction site Model data frequently utilized on the construction site

Luminary is a challenging hybrid structure for designers, builders and fabricators alike. The challenges have to do with its complexity and the number of elevations, tall building

Methods and the changing wishes of future residents. Success relates to model-based design and collaboration as well as sharing and utilizing model data on site.

A-Insinoorit Sunittelu is carrying out the structural and building unit design of the project. In addition, they delivered geotechnical design including level design and acoustical design including studies of the audio-technical qualities of the stairways. Precast manufacturer Parma participated in model-based cooperation. The IFC 
models and architectural DWG files from other design disciplines were used as references. The Tekla model produced 3070 drawings in total, of which 1550 were for the precast units. Including the Delta beams, hollow core slabs and floor slabs, the project consists of 2580 units, and the Tekla model contains about 220,000 objects.

The plot will be filled to its capacity, due to which the supports and foundations have posed a challenge. Existing buildings on both sides, street elevations and other city infrastructure had to be taken into consideration in the design. The foundations feature various levels, and the cast-in-place sublevels include plenty of reinforcing bars, equipment, penetrations and connections to municipal infrastructure. Basic Tekla modeling and reinforcing bar tools have been used in the modeling in addition to equipment and casting components, such as joint reinforcements.

A special challenge was to match the two different frame solutions; the business section contains a beam-to-column frame while the apartments include load-bearing internal walls. Regarding the CIP contractor, the casting areas were large with many complex shapes, and there was very little storage space on site. Using the models, it was easier to manage the contract as a whole.

Precast manufacturing challenges were mainly due to the strict tolerance requirements for tall buildings. The windows and doors were installed on the units at the factory, and the precast detailer modelled these parts to Tekla in order to manage the weights and center of gravity using the model. Design was more demanding than usual, partially because the wall elements include large openings and loads. However, it was possible to model all units in detail and produce the drawings directly from the model.

The units' electrical equipment was modelled based on the electrical designer's IFC files. The temporary bracing and steel connections of thin-shell panels were modelled in exceptional detail. Also, additional joint parts, like welding plates, have been modelled. Attention has been paid to the occupational safety of the units already during the design, and the contractor-modelled solutions for fall protection. The structural designer modelled the transportation supports of the door openings plus all the other structures needed to support the units of various shapes.
The support points for the cranes and elevators for construction work were also modelled. The safety of the lifts was ensured carefully, and the model was utilized as a visual aid. Perhaps the most particular work-time structure modelled is a temporary support for the crane on the 15 th floor as requested by the builders. They needed to find out the exact centre of gravity of the very complex support in order to design and install it safely and accurately horizontally.

Model-based design and visual presentation is a normal design method for the architect. In Luminary, the model was also utilized to check the views from the apartments and balconies to the surrounding area. The project was successful because the design team was able to work seamlessly together, produce high quality, and accurate models. Thanks to Tekla Model Sharing, the builders and the precast manufacturer were able to access the model in real time in a more accurate and visual form compared to a shared IFC model. Among other benefits, accurate modelling has enabled obtaining quantities directly from the model.

On site, managers and employees have used the model in a versatile way alike, for example to visually aid installations and carpentry, at the so-called BIM kiosk. In this project, for the first time, the positions on site were measured based on the structural model, which made work significantly easier and faster. The Tekla model was used on mobile devices with the help of Field3D, and so far, it has been shared among 50 users.

\section{CODES AND STANDARDS}

Sociological changes, new technology in industry and commerce, new building codes, other new laws and regulations, inflationary economies of nations, and advances in building technology place an ever-increasing burden on building designers and constructors. They need more and more knowledge and skill to cope with the demands placed on them.

The public continually demands more complex buildings than in the past. They must serve more purposes, last longer, and require less maintenance and repair. As in the past, they must look attractive. Yet, both building construction and operating costs must be kept within acceptable limits or new construction will cease. 
To meet this challenge successfully, continual improvements in building design and construction must be made. Building designers and constructors should be alert to these advances and learn how to apply them skillfully.

One advance of note to building design is the adaptation of operations research, or systems design, developed around the middle of the twentieth century and originally applied with noteworthy results to design of machines and electronic equipment. In the past, design of a new building was mainly an imitation of the design of an existing building. Innovations were often developed fortuitously and by intuition and were rare occurrences. In contrast, systems design encourages innovation. It is a precise procedure that guides creativity toward the best decisions. As a result, it can play a significant role in meeting the challenges posed by increasing building complexity and costs. The basic principles of systems design are presented in this section.

This chapter covers the applicable codes and standards for the analysis, design and detailing of MSB.

1. IS: 1893(Part-1) - 2002, Criteria for Earthquake Resistant Design of Structures: General provisions and Buildings.

2. IS: 875 (Part-1\&2)-1987, Code Of Practice for Design Loads (Other Than Earthquakes) For Buildings \& Structures - Imposed loads.

3. IS: 875 (Part-3)-1987, Code Of Practice for Design Loads (Other Than Earthquakes) For Buildings \& Structures - Wind loads.

4. IS: 456-2000, Code Of Practice For Plain \& Reinforced Concrete.

5. IS 13920 : 1993 - Indian Standard - Ductile Detailing of Reinforced Concrete Structures Subjected to Seismic Forces - Code of practice.

\section{MSB Design in Tekla structures:}

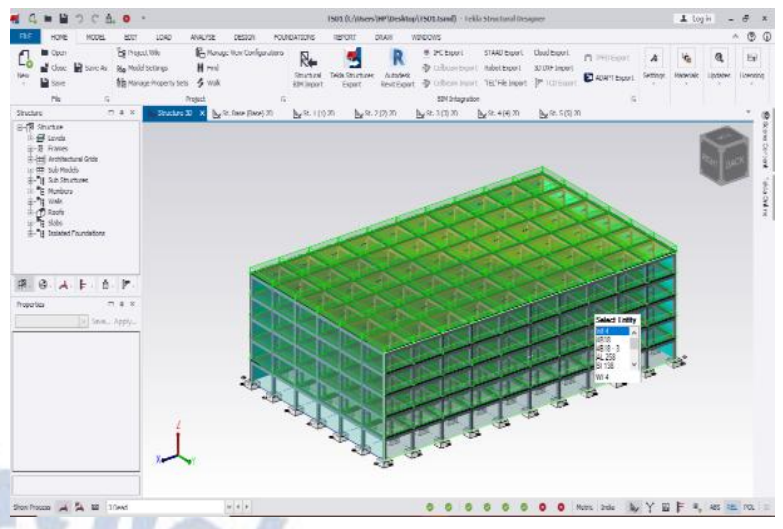

Material Listing:

Structure

Cast-in-place Concrete Beams

\begin{tabular}{|c|c|c|c|c|c|c|c|}
\hline $\begin{array}{c}\text { Sectio } \\
\mathbf{n}\end{array}$ & $\begin{array}{c}\text { Sec } \\
\text { tio }\end{array}$ & $\begin{array}{c}\text { Gra } \\
\text { de }\end{array}$ & $\begin{array}{c}\text { No } \\
\text {. }\end{array}$ & $\begin{array}{c}\text { Le } \\
\text { ng }\end{array}$ & $\begin{array}{c}\text { Mass } \\
{[\mathbf{k g}]}\end{array}$ & $\begin{array}{c}\text { Surface } \\
\text { Area }\end{array}$ & $\begin{array}{c}\text { Volu } \\
\text { me }\end{array}$ \\
\hline Rectan & 450 & M & 10 & 5. & 29270 & 1020.60 & 114. \\
\hline & 450 & M & 72 & 5. & 19513 & 680.400 & 76.5 \\
\hline & 450 & $\mathrm{M}$ & 30 & 5. & 85178 & 2970.00 & 334. \\
\hline & 450 & $\mathrm{M}$ & 20 & 5. & 56785 & 1980.00 & 222. \\
\hline & Tot & & $\mathbf{6 8}$ & & $\mathbf{1 . 9 0 7}$ & $\mathbf{6 6 5 1 . 0 0}$ & $\mathbf{7 4 8 .}$ \\
\hline
\end{tabular}

Cast-in-place Concrete Columns

\begin{tabular}{|c|l|l|l|l|l|l|l|}
\hline $\begin{array}{c}\text { Sectio } \\
\mathbf{n}\end{array}$ & $\begin{array}{l}\text { Secti } \\
\text { on }\end{array}$ & $\begin{array}{l}\text { Gra } \\
\text { de }\end{array}$ & $\begin{array}{l}\text { No } \\
\text {. }\end{array}$ & $\begin{array}{l}\text { Leng } \\
\text { th }\end{array}$ & $\begin{array}{l}\text { Mass } \\
\text { [kg] }\end{array}$ & $\begin{array}{l}\text { Surface } \\
\text { Area }\end{array}$ & $\begin{array}{l}\text { Vol } \\
\text { um }\end{array}$ \\
\hline Rectan & $500 \mathrm{x}$ & $\mathrm{M}$ & 38 & 3.20 & 7851 & 2464.0 & 308 \\
\hline & Total & & $\mathbf{3 8}$ & & $\mathbf{7 8 5 1}$ & $\mathbf{2 4 6 4 . 0}$ & $\mathbf{3 0 8}$ \\
\hline
\end{tabular}

Slabs/Mats

\begin{tabular}{|c|c|l|c|l|}
\hline $\begin{array}{c}\text { Na } \\
\text { me }\end{array}$ & $\begin{array}{c}\text { Mass } \\
{[\mathbf{k g}]}\end{array}$ & $\begin{array}{l}\text { Gross } \\
\text { Surface }\end{array}$ & $\begin{array}{c}\text { Net } \\
\text { Surface }\end{array}$ & $\begin{array}{c}\text { Volum } \\
\mathbf{e}\end{array}$ \\
\hline S 1 & 825970 & 2160.000 & 2160.000 & 324.0 \\
\hline S 2 & 825970 & 2160.000 & 2160.000 & 324.0 \\
\hline S 3 & 825970 & 2160.000 & 2160.000 & 324.0 \\
\hline S 4 & 825970 & 2160.000 & 2160.000 & 324.0 \\
\hline S 5 & 825970 & 2160.000 & 2160.000 & 324.0 \\
\hline Tot & $\mathbf{4 . 1 3 E}+$ & $\mathbf{1 0 8 0 0 . 0 0}$ & $\mathbf{1 0 8 0 0 . 0 0}$ & $\mathbf{1 6 2 0 . 0}$ \\
\hline
\end{tabular}

Reinforcement

Beams

Loose Bars

\begin{tabular}{|c|c|c|c|}
\hline Typ & $\begin{array}{l}\text { Unit } \\
\text { Mass }\end{array}$ & $\begin{array}{c}\text { Total } \\
\text { Lenoth }\end{array}$ & $\begin{array}{l}\text { Total } \\
\text { Mass }\end{array}$ \\
\hline T10 & 0.617 & & \\
\hline $\mathrm{T} 12$ & 0.888 & 1021 & 9074.65 \\
\hline T16 & 1.58 & 5020 & 7932.07 \\
\hline $\mathrm{T} 20$ & 2.470 & 18721.60 & 46242.3 \\
\hline $\mathrm{T} 25$ & 3.850 & 9585.200 & 369 \\
\hline
\end{tabular}

Columns

Loose Bars

\begin{tabular}{|l|c|c|c|}
\hline Typ & $\begin{array}{c}\text { Unit } \\
\text { Mass }\end{array}$ & $\begin{array}{c}\text { Total } \\
\text { Lenoth }\end{array}$ & $\begin{array}{c}\text { Total } \\
\text { Mass }\end{array}$ \\
\hline T8 & 0.395 & 13489.95 & 5328.53 \\
\hline T20 & 2.470 & 4466.000 & 11031.0 \\
\hline T25 & 3.850 & 2479.400 & 9545.69 \\
\hline T32 & 6.310 & 10333.40 & 65203.7 \\
\hline \multicolumn{3}{|r}{ Total } & $\mathbf{9 1 1 0 8}$ \\
\hline
\end{tabular}


Slabs/Mats

Loose Bars

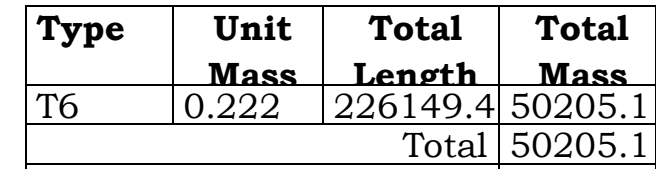

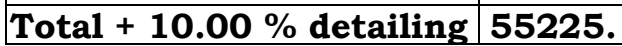

Concrete

\begin{tabular}{|l|c|l|l|c|}
\hline $\begin{array}{l}\text { Grad } \\
\mathbf{e}\end{array}$ & $\begin{array}{c}\text { Mass } \\
{[\mathbf{k g}]}\end{array}$ & $\begin{array}{l}\text { Gross } \\
\text { Surface }\end{array}$ & $\begin{array}{l}\text { Net } \\
\text { Surface }\end{array}$ & $\begin{array}{c}\text { Volu } \\
\text { me }\end{array}$ \\
\hline M 25 & $1.93 \mathrm{E}+$ & 6454.600 & 6454.60 & 756.9 \\
\hline M 20 & $4.893 \mathrm{E}$ & 13460.400 & 13460.4 & 1919. \\
\hline M 30 & 476997 & 914.760 & 914.760 & 187.1 \\
\hline Tota & $\mathbf{7 . 3 E}+\mathbf{0}$ & $\mathbf{2 0 8 2 9 . 7 6}$ & $\mathbf{2 0 8 2 9 . 7}$ & $\mathbf{2 8 6 3}$ \\
\hline \multicolumn{4}{|l}{ Total } \\
\hline
\end{tabular}

\begin{tabular}{|c|c|c|c|c|}
\hline $\begin{array}{c}\text { Mat } \\
\text { aria1 }\end{array}$ & $\begin{array}{c}\text { Mass } \\
\text { [locl }\end{array}$ & $\begin{array}{c}\text { Gross } \\
\text { aurfana }\end{array}$ & $\begin{array}{c}\text { Net } \\
\text { curfana }\end{array}$ & $\begin{array}{c}\text { Volu } \\
\text { ma }\end{array}$ \\
\hline Conc & $7.3 \mathrm{E}+$ & 20829.76 & 20829.760 & 2863. \\
\hline
\end{tabular}

\begin{tabular}{|l|l|l|l|l|}
\hline Tota & $7.3 \mathrm{E}+20829.76$ & 20829.760 & 2863 \\
\hline
\end{tabular} Reinforcement

\begin{tabular}{|l|c|}
\hline Type & $\begin{array}{c}\text { Total } \\
\text { Mass }\end{array}$ \\
\hline Beams & 154502. \\
\hline Columns & 91108.9 \\
\hline Slabs $/$ Mats & 50205.1 \\
\hline Total + 10.00 \% & $\mathbf{3 0 0 8 3 7}$ \\
\hline
\end{tabular}

\section{Methodology/Procedure}

Step-1: Initial setup of Standard Codes and Country codes.

Step-2: Creation of Grids points \&Generation structure.

After getting opened with TEKLA we select a new model and a window appears where we had entered the grid dimensions and storey dimensions of our building.

\section{Step-3: Defining of property}

Here we had first defined material property by selecting define menu material properties. We add a new material for our structural components (beams, columns, slabs) by giving the specified details in defining. After that we define section size by selecting frame sections as shown below \& added the required section for beams, columns etc.

\section{Step-4 Assigning of Property}

After defining property we draw the structural components using command menu. Create a grids for beams and create a columns in region for columns by which property assigning is completed for beams and columns.
By keeping the selection at the base of the structure and selecting all columns, we assigned supports by going to assign menu joint/frame Restraints (supports) fixed.

\section{Step-6 defining of loads}

In TEKLA all the load considerations are first defined and then assigned. The loads in TEKLA are defined as using static load cases command in define menu.

\section{Step-7 Assigning of Dead loads}

After defining all loads. Dead loads are assigned for external walls, internal walls in staad but TEKLA automatically taken care by the software i.e., inbuilt

\section{Step-8 Assigning of Live loads}

Live loads are assigned for the entire structure including floor finishing.

\section{Step-9 Assigning of wind loads}

Wind loads are defined and assigned as per IS 875: 1987 PART-3 by giving the wind speed and wind angle. But since this is a G+5 Multi-stored Building and assigning of wind loads or earth quake loads.

\section{Step-10 Assigning of Seismic loads}

Seismic loads are defined and assigned as per IS 1893:2002 by giving zone, soil type, and response reduction factor in $\mathrm{X}$ and $\mathrm{Y}$ directions. But since this is a G+5Multi-stored Building and assigning Seismic loads.

\section{Step-11 Assigning of load combinations}

Using load combinations command in define menu 1.5 times of dead load and live load will be taken as mentioned in above.

\section{Step-12 Analysis}

After the completion of all the above steps we have performed the analysis and checked for errors.

\section{Step-13 Design}

After the completion of analysis we had performed concrete design on the structure as per IS 456:2000. TEKLA performs the design for every structural element.

\section{Step-5 Assigning of Supports}


Load combination factors

\begin{tabular}{|l|c|c|c|c|}
\hline \multirow{2}{*}{ Load combination } & \multicolumn{2}{|c|}{ Off } & \multicolumn{2}{|c|}{ On } \\
& \multicolumn{2}{|c|}{ Off } \\
\hline $\begin{array}{l}\text { Dead + Live } \\
\text { (Strength) }\end{array}$ & \multicolumn{2}{|c|}{ On } & \multicolumn{2}{|c|}{ Off } \\
\hline
\end{tabular}

Element Loads

\begin{tabular}{|c|c|c|c|l|}
\hline $\begin{array}{c}\text { Elem } \\
\text { ent }\end{array}$ & $\begin{array}{c}\text { Load } \\
\text { case }\end{array}$ & $\begin{array}{c}\text { Load } \\
\text { Type }\end{array}$ & $\begin{array}{c}\text { Orientat } \\
\text { ion }\end{array}$ & $\begin{array}{l}\text { Descri } \\
\text { ption }\end{array}$ \\
\hline 1 & Dead & UDL & GlobalZ & $\begin{array}{l}18 \\
\mathrm{kN} / \mathrm{m}\end{array}$ \\
\hline 2 & Dead & UDL & GlobalZ & $\begin{array}{l}18 \\
\mathrm{kN} / \mathrm{m}\end{array}$ \\
\hline 1 & Live & UDL & GlobalZ & $\begin{array}{l}15 \\
\mathrm{kN} / \mathrm{m}\end{array}$ \\
\hline 2 & Live & UDL & GlobalZ & $\begin{array}{l}15 \\
\mathrm{kN} / \mathrm{m}\end{array}$ \\
\hline
\end{tabular}

Results

Reactions

Load combination: Dead + Live (Strength)

\begin{tabular}{|c|c|c|c|}
\hline \multirow[t]{2}{*}{ Node } & \multicolumn{2}{|c|}{ Force } & Moment \\
\hline & $\begin{array}{c}\text { Fx } \\
(\mathbf{k N})\end{array}$ & $\begin{array}{c}\mathbf{F z} \\
(\mathbf{k N})\end{array}$ & $\begin{array}{c}\text { My } \\
\text { (kNm) }\end{array}$ \\
\hline 1 & 0 & 127.2 & -117.8 \\
\hline 2 & 0 & 312.4 & 0 \\
\hline 3 & 0 & 107.6 & 0 \\
\hline
\end{tabular}

Load combination: Dead + Live (Strength On

\begin{tabular}{|c|c|c|c|}
\hline \multirow[t]{2}{*}{ Node } & \multicolumn{2}{|c|}{ Force } & \multirow{2}{*}{$\begin{array}{c}\text { Moment } \\
\text { My } \\
(\mathbf{k N m})\end{array}$} \\
\hline & $\begin{array}{l}\text { Fx } \\
(\mathbf{k N})\end{array}$ & $\begin{array}{c}\mathrm{Fz} \\
(\mathbf{k N})\end{array}$ & \\
\hline 1 & 0 & 144.8 & -152.6 \\
\hline 2 & 0 & 200.3 & 0 \\
\hline 3 & 0 & 36.5 & 0 \\
\hline
\end{tabular}

Load combination: Dead + Live (Strength Off

\begin{tabular}{|c|c|c|c|}
\hline Node & \multicolumn{2}{|c|}{ Force } & Moment \\
& Fx & $\mathbf{F z}$ & $\mathbf{M y}$ \\
& $(\mathbf{k N})$ & $(\mathbf{k N})$ & $(\mathbf{k N m})$ \\
\hline 1 & 0 & 32.7 & -11.7 \\
\hline 2 & 0 & 235.4 & 0 \\
\hline 3 & 0 & 113.5 & 0 \\
\hline
\end{tabular}

On)
Load combination: Dead + Live (Strength On On

Off)

\begin{tabular}{|c|c|c|c|}
\hline \multirow[t]{2}{*}{ Node } & \multicolumn{2}{|c|}{ Force } & \multirow{2}{*}{$\begin{array}{c}\text { Moment } \\
\text { My } \\
\text { (kNm) }\end{array}$} \\
\hline & $\begin{array}{c}F x \\
(k N)\end{array}$ & $\begin{array}{c}\mathbf{F z} \\
(\mathbf{k N})\end{array}$ & \\
\hline 1 & 0 & 127.2 & -117.8 \\
\hline 2 & 0 & 312.4 & 0 \\
\hline 3 & 0 & 107.6 & 0 \\
\hline
\end{tabular}

Load combination: Dead + Live (Strength On Off

\begin{tabular}{|c|c|c|c|}
\hline Node & \multicolumn{2}{|c|}{ Force } & Moment \\
& $\mathbf{F x}$ & $\mathbf{F z}$ & $\mathbf{M y}$ \\
& $(\mathbf{k N})$ & $(\mathbf{k N})$ & $(\mathbf{k N m})$ \\
\hline 1 & 0 & 144.8 & -152.6 \\
\hline 2 & 0 & 200.3 & 0 \\
\hline 3 & 0 & 36.5 & 0 \\
\hline
\end{tabular}

Load combination: Dead + Live (Strength Off On

\begin{tabular}{|c|c|c|c|}
\hline Node & \multicolumn{2}{|c|}{ Force } & Moment \\
& $\mathbf{F x}$ & $\mathbf{F z}$ & $\mathbf{M y}$ \\
& $(\mathbf{k N})$ & $(\mathbf{k N})$ & $(\mathbf{k N m})$ \\
\hline 1 & 0 & 32.7 & -11.7 \\
\hline 2 & 0 & 235.4 & 0 \\
\hline 3 & 0 & 113.5 & 0 \\
\hline
\end{tabular}

Element end forces

Load combination: Dead + Live (Strength)

\begin{tabular}{|c|c|c|c|c|c|}
\hline $\begin{array}{c}\text { Elem } \\
\text { ent }\end{array}$ & $\begin{array}{c}\text { Leng } \\
\text { th } \\
(\mathbf{m})\end{array}$ & $\begin{array}{c}\text { Nodes } \\
\text { Start/ } \\
\text { End }\end{array}$ & $\begin{array}{c}\text { Axial } \\
\text { force } \\
\mathbf{( k N )}\end{array}$ & $\begin{array}{c}\text { Shear } \\
\text { force } \\
\mathbf{( k N )}\end{array}$ & $\begin{array}{c}\text { Mome } \\
\mathbf{n t} \\
\mathbf{( k N m})\end{array}$ \\
\hline \multirow{2}{*}{1} & \multirow{2}{*}{6} & 1 & 0 & -127.2 & 117.8 \\
\cline { 3 - 6 } & & 2 & 0 & -146.4 & -175.3 \\
\hline 2 & \multirow{2}{*}{6} & 2 & 0 & -166 & 175.3 \\
\cline { 3 - 6 } & & 3 & 0 & -107.6 & 0 \\
\hline
\end{tabular}

Load combination: Dead + Live (Strength On Off)

\begin{tabular}{|c|c|c|c|c|c|}
\hline $\begin{array}{c}\text { Elem } \\
\text { ent }\end{array}$ & $\begin{array}{c}\text { Leng } \\
\text { th } \\
(\mathbf{m})\end{array}$ & $\begin{array}{c}\text { Nodes } \\
\text { Start/E } \\
\text { nd }\end{array}$ & $\begin{array}{c}\text { Axial } \\
\text { force } \\
\mathbf{( k N )}\end{array}$ & $\begin{array}{c}\text { Shear } \\
\text { force } \\
\mathbf{( k N )}\end{array}$ & $\begin{array}{c}\text { Mome } \\
\mathbf{n t} \\
\mathbf{( k N m})\end{array}$ \\
\hline 1 & \multirow{2}{*}{6} & 1 & 0 & -144.8 & 152.6 \\
\cline { 3 - 6 } & & 2 & 0 & -128.8 & -104.8 \\
\hline 2 & \multirow{2}{*}{6} & 2 & 0 & -71.5 & 104.8 \\
\cline { 3 - 6 } & & 3 & 0 & -36.5 & 0 \\
\hline
\end{tabular}


Load combination: Dead + Live (Strength Off

On)

\begin{tabular}{|c|c|c|c|c|c|}
\hline $\begin{array}{c}\text { Eleme } \\
\mathbf{n t}\end{array}$ & $\begin{array}{c}\text { Lengt } \\
\mathbf{h}\end{array}$ & Nodes & $\begin{array}{l}\text { Axia } \\
\mathbf{1} \\
\text { forc } \\
\mathbf{e}\end{array}$ & $\begin{array}{l}\text { Shear } \\
\text { force }\end{array}$ & Moment \\
& $\mathbf{( m )}$ & $\begin{array}{c}\text { Start/E } \\
\mathbf{n d}\end{array}$ & $\begin{array}{l}\mathbf{( k N )} \\
\mathbf{( k N )}\end{array}$ & $\mathbf{( k N m )}$ \\
\hline 1 & \multirow{2}{*}{6} & 1 & 0 & -32.7 & 11.7 \\
\cline { 3 - 6 } & & 2 & 0 & -75.3 & -139.6 \\
\hline 2 & \multirow{2}{*}{6} & 2 & 0 & -160.1 & 139.6 \\
\cline { 3 - 6 } & & 3 & 0 & -113.5 & 0 \\
\hline
\end{tabular}

Load combination: Dead + Live (Strength On On Off)

\begin{tabular}{|c|c|c|c|c|c|}
\hline $\begin{array}{c}\text { Elem } \\
\text { ent }\end{array}$ & Length & Nodes & $\begin{array}{c}\text { Axial } \\
\text { force } \\
\text { (m) }\end{array}$ & $\begin{array}{c}\text { Shear } \\
\text { Start/ } \\
\text { force } \\
\text { End }\end{array}$ & $\begin{array}{c}\text { Momen } \\
\mathbf{t} \\
\mathbf{( k N )} \\
\mathbf{( k N m )}\end{array}$ \\
\hline 1 & 6 & 1 & 0 & -127.2 & 117.8 \\
\cline { 3 - 6 } & \multirow{2}{*}{6} & 2 & 0 & -146.4 & -175.3 \\
\hline \multirow{2}{*}{6} & 2 & 0 & -166 & 175.3 \\
\cline { 3 - 6 } & & 3 & 0 & -107.6 & 0 \\
\hline
\end{tabular}

Load combination: Dead + Live (Strength On Off On)

\begin{tabular}{|c|c|c|c|c|c|}
\hline $\begin{array}{c}\text { Elem } \\
\text { ent }\end{array}$ & Length & Nodes & $\begin{array}{c}\text { Axial } \\
\text { force } \\
\text { (m) }\end{array}$ & $\begin{array}{c}\text { Shear } \\
\text { Start/ } \\
\text { force } \\
\text { End }\end{array}$ & $\begin{array}{c}\text { Momen } \\
\mathbf{( k N )} \\
\mathbf{( k N m )}\end{array}$ \\
\hline 1 & 6 & 1 & 0 & -144.8 & 152.6 \\
\cline { 3 - 6 } & & 2 & 0 & -128.8 & -104.8 \\
\hline 2 & 6 & 2 & 0 & -71.5 & 104.8 \\
\cline { 3 - 6 } & & 3 & 0 & -36.5 & 0 \\
\hline
\end{tabular}

Load combination: Dead + Live (Strength Off On

On)

\begin{tabular}{|c|c|c|c|c|c|}
\hline $\begin{array}{c}\text { Elem } \\
\text { ent }\end{array}$ & Length & Nodes & $\begin{array}{c}\text { Axial } \\
\text { force } \\
\text { (m) }\end{array}$ & $\begin{array}{c}\text { Shear } \\
\text { Start/ } \\
\text { force } \\
\text { End }\end{array}$ & $\begin{array}{c}\text { Momen } \\
\mathbf{t} \\
(\mathbf{k N})\end{array}$ \\
\hline \multirow{2}{*}{1} & \multirow{2}{*}{6} & 1 & 0 & -32.7 & 11.7 \\
\cline { 3 - 6 } & \multirow{2}{*}{6} & 2 & 0 & -75.3 & -139.6 \\
\hline 2 & \multirow{2}{*}{6} & 2 & 0 & -160.1 & 139.6 \\
\cline { 3 - 6 } & & 3 & 0 & -113.5 & 0 \\
\hline
\end{tabular}

\section{Forces}

Strength combinations - Moment envelope (kNm)

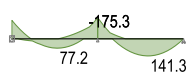

Strength combinations - Shear envelope $(\mathrm{kN})$

144.

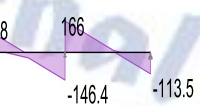

Member results

Envelope - Strength combinations

\begin{tabular}{|c|c|c|c|c|c|}
\hline \multirow{2}{*}{$\begin{array}{c}\begin{array}{c}\text { Mem } \\
\text { ber }\end{array} \\
\text { Beam }\end{array}$} & \multirow{2}{*}{$\begin{array}{c}\text { Positi } \\
\text { on } \\
\text { (m) }\end{array}$} & \multicolumn{2}{|c|}{$\begin{array}{c}\text { Shear force } \\
(\mathbf{k N})\end{array}$} & \multicolumn{2}{|c|}{$\begin{array}{l}\text { Moment } \\
\text { (kNm) }\end{array}$} \\
\hline & & $\begin{array}{c}166 \text { (max } \\
\text { abs) }\end{array}$ & $\begin{array}{c}-146 . \\
4\end{array}$ & -104.8 & $\begin{array}{c}-175.3 \\
\text { (min) }\end{array}$ \\
\hline & 9.51 & 8.3 & 0 & $\begin{array}{l}141.3 \\
(\max )\end{array}$ & 35.2 \\
\hline
\end{tabular}

Dead + Live (Strength) - Moment (kNm)

$$
\frac{-117.8 \quad-175.3}{59.7 \quad 126.9}
$$

Dead + Live (Strength) - Shear (kN)

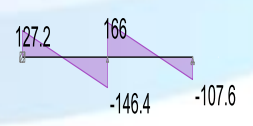

Member results

Load combination: Dead + Live (Strength)

\begin{tabular}{|c|c|c|c|c|}
\hline $\begin{array}{c}\text { Memb } \\
\text { er }\end{array}$ & $\begin{array}{c}\text { Positi } \\
\text { on } \\
(\mathbf{m})\end{array}$ & \multicolumn{2}{|c|}{ Shear force } & \multicolumn{2}{|c|}{ Moment } \\
& $\mathbf{( k N )}$ & \multicolumn{2}{|c|}{$\mathbf{( k N m )}$} \\
\hline Beam & 6 & $\begin{array}{c}166(\max \\
\text { abs })\end{array}$ & $\begin{array}{c}-146 . \\
4\end{array}$ & $\begin{array}{c}-175.3 \\
(\min )\end{array}$ \\
\cline { 2 - 6 } & 9.641 & 0 & & $\begin{array}{c}126.9 \\
(\max )\end{array}$ \\
\hline
\end{tabular}


Dead + Live (Strength On Off) - Moment (kNm)

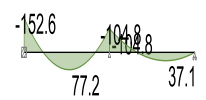

Dead + Live (Strength On Off) - Shear (kN)

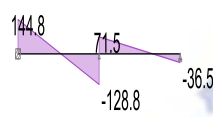

Member results

Load combination: Dead + Live (Strength On

Off)

\begin{tabular}{|c|c|c|c|c|c|}
\hline $\begin{array}{c}\text { Memb } \\
\text { er }\end{array}$ & $\begin{array}{l}\text { Positi } \\
\text { on } \\
\text { (m) }\end{array}$ & Shear for & & $\left(\mathrm{kN}_{\mathbf{1}}\right.$ & \\
\hline Beam & 0 & $\begin{array}{c}144.8 \text { (max } \\
\mathrm{abs})\end{array}$ & & $\begin{array}{c}-152.6 \\
\text { (min) }\end{array}$ & \\
\hline \multirow[t]{2}{*}{ * } & 3.175 & 0 & & $\begin{array}{c}77.2 \\
\text { (max) }\end{array}$ & \\
\hline & 6 & 71.5 & $\begin{array}{c}-128 \\
.8\end{array}$ & -104.8 & \\
\hline
\end{tabular}

Dead + Live (Strength Off On) - Moment (kNm)

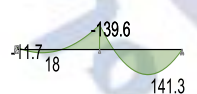

Dead + Live (Strength Off On) - Shear (kN)

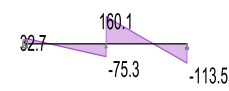

Member results

Load combination: Dead + Live (Strength Off

On)

\begin{tabular}{|c|c|c|c|c|}
\hline $\begin{array}{c}\text { Memb } \\
\text { er }\end{array}$ & $\begin{array}{c}\text { Positio } \\
\text { n } \\
\text { (m) }\end{array}$ & $\begin{array}{r}\text { Shear f } \\
(\mathbf{k N})\end{array}$ & rce & $\begin{array}{r}\text { Momen } \\
\text { (kNm) }\end{array}$ \\
\hline Beam & 6 & $\begin{array}{c}160.1 \\
(\max a b s)\end{array}$ & -75.3 & $\begin{array}{c}-139.6 \\
(\mathrm{~min})\end{array}$ \\
\hline & 9.51 & 0 & & $\begin{array}{l}141.3 \\
(\max )\end{array}$ \\
\hline & 12 & -113.5 & & 0 \\
\hline
\end{tabular}

Dead + Live (Strength On On Off) - Moment $(\mathbf{k N m})$

$$
\frac{-117.8 \quad-175.3}{59.7 \quad 126.9}
$$

Dead + Live (Strength On On Off) - Shear (kN)

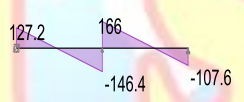

\section{Member results}

Load combination: Dead + Live (Strength On

On

Off)

\begin{tabular}{|c|c|c|c|c|}
\hline $\begin{array}{c}\text { Memb } \\
\text { er }\end{array}$ & $\begin{array}{c}\text { Positio } \\
\text { n } \\
\text { (m) }\end{array}$ & $(\mathrm{kN})$ & rce & $\begin{array}{l}\text { Moment } \\
\text { (kNm) }\end{array}$ \\
\hline \multirow[t]{2}{*}{ Beam } & 6 & $\begin{array}{c}166(\max \\
\text { abs) }\end{array}$ & $\begin{array}{c}-146 . \\
4\end{array}$ & $\begin{array}{c}-175.3 \\
\text { (min) }\end{array}$ \\
\hline & 9.641 & 0 & 8 & $\begin{array}{l}126.9 \\
(\max )\end{array}$ \\
\hline
\end{tabular}

Dead + Live (Strength On Off On) - Moment $(\mathbf{k N m})$

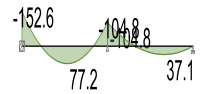


Dead + Live (Strength On Off On) - Shear (kN)

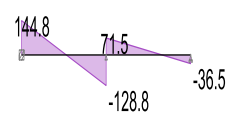

\section{Member results}

Load combination: Dead + Live (Strength On Off

\begin{tabular}{|c|c|c|c|c|c|}
\hline $\begin{array}{c}\text { Membe } \\
\mathbf{r}\end{array}$ & $\begin{array}{c}\text { Positi } \\
\text { on } \\
(\mathbf{m})\end{array}$ & \multicolumn{2}{|c|}{ Shear force } & \multicolumn{2}{|c|}{ Moment } \\
& \multicolumn{2}{|c|}{$(\mathbf{k N})$} & \multicolumn{2}{|c|}{$\mathbf{( \mathbf { k N m } )}$} \\
\hline Beam & 0 & $\begin{array}{c}144.8(\max \\
\mathrm{abs})\end{array}$ & $\begin{array}{c}-152.6 \\
(\min )\end{array}$ & \\
\cline { 2 - 6 } & 3.175 & 0 & & $77.2(\max )$ & \\
\cline { 2 - 6 } & 6 & 71.5 & -128 & -104.8 & \\
8 & & & \\
\hline
\end{tabular}

Dead + Live (Strength Off On On) - Moment (kNm)

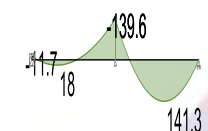

Dead + Live (Strength Off On On) - Shear (kN)

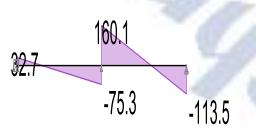

\section{Member results}

Load combination: Dead + Live (Strength Off On

\begin{tabular}{|c|c|c|c|c|}
\hline $\begin{array}{c}\text { Memb } \\
\text { er }\end{array}$ & $\begin{array}{c}\text { Positio } \\
\mathbf{n} \\
(\mathbf{m})\end{array}$ & \multicolumn{2}{|c|}{ Shear force } & Moment \\
\hline Beam & 6 & $\begin{array}{c}160.1(\max ) \\
\text { abs) }\end{array}$ & -75. & $\begin{array}{c}-139.6 \\
(\mathbf{k N m})\end{array}$ \\
\hline
\end{tabular}

\begin{tabular}{|c|c|c|c|}
\hline \multirow[t]{2}{*}{$\begin{array}{c}\text { Memb } \\
\text { er }\end{array}$} & \multirow{2}{*}{\begin{tabular}{|c|}
$\begin{array}{c}\text { Positio } \\
\mathbf{n}\end{array}$ \\
(m)
\end{tabular}} & $\begin{array}{c}\text { Shear force } \\
\text { (kN) }\end{array}$ & $\begin{array}{r}\text { Momen } \\
(\mathbf{k N m})\end{array}$ \\
\hline & & 0 & $\begin{array}{l}141.3 \\
(\max )\end{array}$ \\
\hline & 12 & -113.5 & 0 \\
\hline
\end{tabular}

Beam loading and results for major axis:
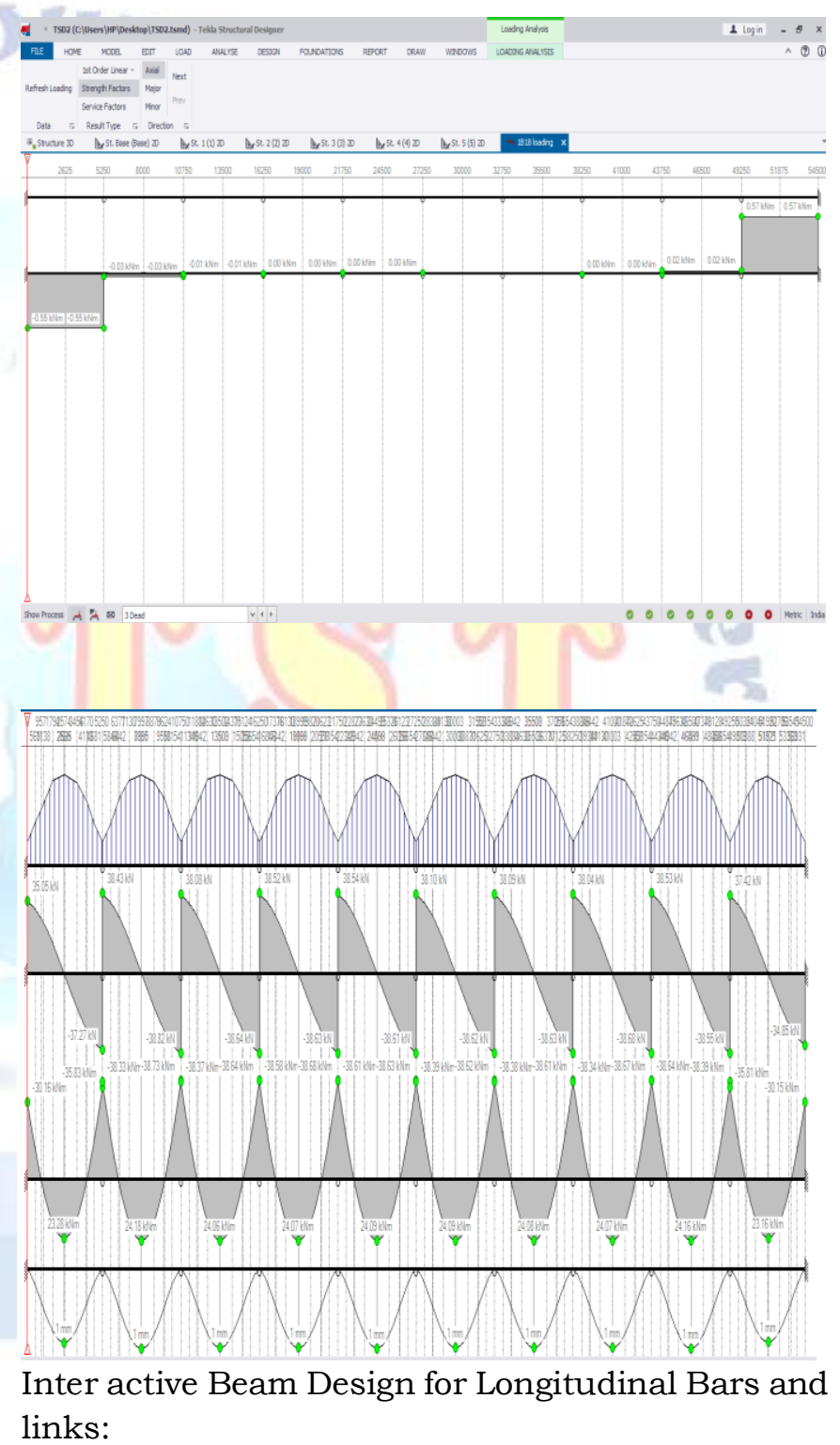

Inter active Beam Design for Longitudinal Bars and 

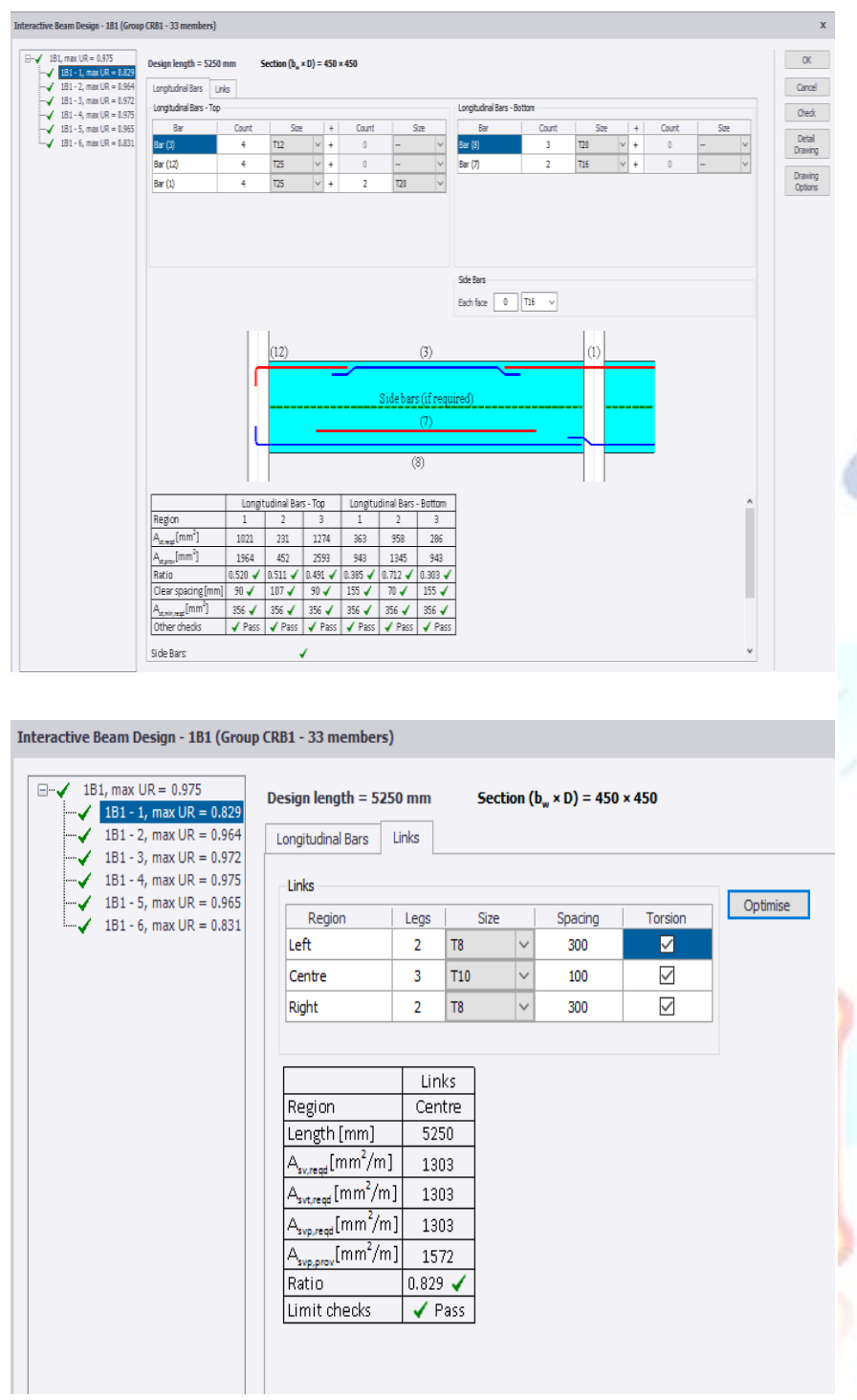

\section{Element Properties:}

Section Properties

\begin{tabular}{|c|c|c|c|c|c|c|c|}
\hline ecti & & $\mathbf{I}_{\mathbf{t}}$ & I major & $I_{\text {minor }}$ & A & $\mathbf{A}_{\|}$ & $\mathbf{A}_{\|}$ \\
\hline & & & & & $\begin{array}{l}\operatorname{lm} \\
202\end{array}$ & 16 & 168 \\
\hline & M & 57755 & 3417 & 3417 & 202 & 168 & 16 \\
\hline & $\mathrm{N}$ & 88028 & & & 250 & 20 & 20 \\
\hline
\end{tabular}

Modification Factors

\begin{tabular}{|l|l|l|l|r|r|r|r|r|r|r|}
\hline Mate & Type & E & G & $\mathbf{I}_{\mathbf{t}}$ & $\mathbf{I}_{\mathbf{m a}}$ & $\mathbf{I}_{\mathbf{m i}}$ & $\mathbf{A}$ & $\mathbf{A}_{\|}$ & $\mathbf{A}_{\|}$ & $\mathbf{t}$ \\
\hline \multirow{2}{*}{$\begin{array}{l}\text { Conc } \\
\text { rete }\end{array}$} & Column & 1.0 & 1.0 & 1.0 & 1.0 & 1.0 & 1.0 & 1.0 & 1.0 & - \\
\cline { 2 - 10 } & Beam & 1.0 & 1.0 & 1.0 & 1.0 & 1.0 & 1.0 & 1.0 & 1.0 & - \\
\hline
\end{tabular}

\section{Beams:}

End 1/End $2+$ Coincident, First-order linear, All combinations

St. 1 (1)

\begin{tabular}{|c|c|c|c|c|c|c|c|c|c|c|c|c|}
\hline $\begin{array}{l}\text { Refer } \\
\text { ence }\end{array}$ & \begin{tabular}{l|}
$\mathbf{S}$ \\
$\mathbf{p}$ \\
$\mathbf{a}$
\end{tabular} & $\begin{array}{l}\text { Se } \\
\text { cti } \\
\text { on }\end{array}$ & $\begin{array}{l}\text { ra } \\
\text { d }\end{array}$ & d & \begin{tabular}{|l|} 
Con \\
ditio \\
$\mathrm{n}$
\end{tabular} & $\begin{array}{l}\text { Combinati } \\
\text { on }\end{array}$ & \begin{tabular}{|c|}
$\mathbf{F}$ \\
$\mathbf{x}$ \\
$\mathbf{L}$ \\
$\mathbf{k}$ \\
\end{tabular} & $\begin{array}{l}\mathbf{y} \\
{[} \\
\mathbf{k}\end{array}$ & $\begin{array}{l}\mathbf{F}_{\mathbf{z}} \\
{[\mathbf{k}} \\
\mathbf{N}]\end{array}$ & $\begin{array}{r}\mathbf{M}_{\mathbf{x}} \\
{[\mathbf{k}} \\
\mathbf{N} \\
\mathbf{m}] \\
\end{array}$ & \begin{tabular}{|r|}
$\mathbf{M}_{\mathbf{y}}$ \\
{$[\mathbf{k N}$} \\
$\mathbf{m}]$
\end{tabular} & $\begin{array}{r}\mathbf{M}_{\mathbf{z}} \\
{[\mathbf{k}} \\
\mathbf{N} \\
\mathbf{m}]\end{array}$ \\
\hline \multirow[t]{20}{*}{ 1B1 } & \multirow{10}{*}{1} & $\begin{array}{l}45 \\
0 x\end{array}$ & \multirow{10}{*}{$\begin{array}{l}\mathrm{M} \\
2 \\
5\end{array}$} & \multirow[t]{10}{*}{1} & $\begin{array}{l}\operatorname{Min} \\
M_{\mathrm{y}}\end{array}$ & $\begin{array}{l}51 \text { (Final) } \\
\mathrm{LS}_{2.4}-1.5 \mathrm{D}+\end{array}$ & \begin{tabular}{|l|}
0. \\
0
\end{tabular} & \begin{tabular}{|l|}
0. \\
0
\end{tabular} & $\begin{array}{l}14 \\
8 .\end{array}$ & \begin{tabular}{|l|}
2. \\
35
\end{tabular} & \begin{tabular}{|l|}
-16 \\
0.3
\end{tabular} & $\begin{array}{l}0 . \\
00\end{array}$ \\
\hline & & $\begin{array}{l}45 \\
0\end{array}$ & & & $\begin{array}{l}\text { Max } \\
M_{x}\end{array}$ & $\begin{array}{l}5551 \\
\text { (Final) } \\
\text { LS }_{2.4-1.5 D+}\end{array}$ & $\begin{array}{l}0 . \\
0 \\
0\end{array}$ & \begin{tabular}{|l|}
0. \\
0 \\
0
\end{tabular} & $\begin{array}{l}14 \\
7 . \\
22 \\
\end{array}$ & $\begin{array}{l}2 . \\
41\end{array}$ & $\begin{array}{l}-15 \\
8.0 \\
5\end{array}$ & $\begin{array}{l}0 . \\
00\end{array}$ \\
\hline & & & & & \begin{tabular}{|l|}
$\operatorname{Max}$ \\
$F_{z}$
\end{tabular} & $\begin{array}{l}6051 \\
\text { (Final) } \\
\mathrm{LS}_{2.4-1.5 \mathrm{D}+}\end{array}$ & \begin{tabular}{|l|}
0. \\
0 \\
0
\end{tabular} & \begin{tabular}{|l|}
0. \\
0 \\
0
\end{tabular} & \begin{tabular}{|l|}
14 \\
8. \\
52
\end{tabular} & \begin{tabular}{|l|}
2. \\
35
\end{tabular} & \begin{tabular}{|l|}
-15 \\
9.6 \\
8
\end{tabular} & $\begin{array}{l}0 . \\
00\end{array}$ \\
\hline & & & & & Min & 68 (Final) & \begin{tabular}{|l|}
0. \\
0
\end{tabular} & 0. & 58 & $\begin{array}{l}0 . \\
89\end{array}$ & $\begin{array}{r}-49 \\
80\end{array}$ & \\
\hline & & & & & $\frac{\mathrm{M}_{\mathrm{x}}}{\mathrm{Min}}$ & $\frac{\mathrm{LS}_{7.1}-0.9 \mathrm{D}+}{114104}$ & $\begin{array}{l}0 \\
0\end{array}$ & \begin{tabular}{|l|}
0 \\
0.
\end{tabular} & $\begin{array}{l}.4 \\
53\end{array}$ & \begin{tabular}{|l|}
89 \\
1.
\end{tabular} & \begin{tabular}{|l|}
.80 \\
-25
\end{tabular} & $\begin{array}{l}00 \\
0 .\end{array}$ \\
\hline & & & & & $\mathrm{F}_{z}$ & (Final) & 0 & 0 & .7 & 55 & .96 & 00 \\
\hline & & & & & $\begin{array}{l}\text { Max } \\
F_{z},\end{array}$ & $\begin{array}{l}40 \text { (Final) } \\
\mathrm{LS}_{2.3}-1.5 \mathrm{D}+\end{array}$ & $\begin{array}{l}0 . \\
0\end{array}$ & $\begin{array}{l}0 . \\
0\end{array}$ & $\begin{array}{l}15 \\
3 .\end{array}$ & \begin{tabular}{|l}
-2. \\
33
\end{tabular} & \begin{tabular}{|l|}
17 \\
6.7 \\
\end{tabular} & $\begin{array}{l}0 . \\
00\end{array}$ \\
\hline & & & & & $\begin{array}{l}\text { Min } \\
M_{x}\end{array}$ & $\begin{array}{l}5551 \\
\text { (Final) } \\
\text { LS }_{2.4-1.5 D+}\end{array}$ & $\begin{array}{l}0 . \\
0 \\
0\end{array}$ & \begin{tabular}{|l|}
0. \\
0 \\
0
\end{tabular} & $\begin{array}{l}12 \\
0 . \\
29\end{array}$ & \begin{tabular}{|l}
-2. \\
41
\end{tabular} & \begin{tabular}{|l|}
93. \\
52
\end{tabular} & $\begin{array}{l}0 . \\
00\end{array}$ \\
\hline & & & & & $\begin{array}{l}\text { Max } \\
M_{x}\end{array}$ & $\begin{array}{l}68 \text { (Final) } \\
\text { LS }_{7.1}-0.9 \mathrm{D}+\end{array}$ & \begin{tabular}{|l|}
0. \\
0
\end{tabular} & \begin{tabular}{|l|}
0. \\
0
\end{tabular} & $\begin{array}{l}60 \\
.9\end{array}$ & $\begin{array}{l}-0 . \\
89\end{array}$ & $\begin{array}{l}58 . \\
73\end{array}$ & $\begin{array}{l}0 . \\
00\end{array}$ \\
\hline & & & & & $\begin{array}{l}\text { Min } \\
F_{z}\end{array}$ & $\begin{array}{l}125115 \\
\text { (Final) }\end{array}$ & \begin{tabular}{|l|}
0. \\
0
\end{tabular} & \begin{tabular}{|l|}
0. \\
0
\end{tabular} & $\begin{array}{l}57 \\
.3\end{array}$ & \begin{tabular}{|l|}
-1. \\
57
\end{tabular} & $\begin{array}{l}40 . \\
34\end{array}$ & \\
\hline & \multirow[t]{10}{*}{2} & \multirow{10}{*}{$\begin{array}{l}45 \\
0 \mathrm{x} \\
45 \\
0\end{array}$} & \multirow[t]{10}{*}{5} & \multirow[t]{5}{*}{1} & $\begin{array}{l}\text { Max } \\
M_{x}\end{array}$ & $\begin{array}{l}4140 \\
\text { (Final) } \\
\text { LS }_{2.3}-1.5 \mathrm{D}+\end{array}$ & $\begin{array}{l}0 . \\
0 \\
0\end{array}$ & \begin{tabular}{|l|}
0. \\
0 \\
0
\end{tabular} & \begin{tabular}{|l}
12 \\
8. \\
93
\end{tabular} & $\begin{array}{l}0 . \\
08\end{array}$ & \begin{tabular}{|l|}
-10 \\
7.6 \\
8
\end{tabular} & $\begin{array}{l}0 . \\
00\end{array}$ \\
\hline & & & & & $\begin{array}{l}\text { Min } \\
\mathrm{M}_{\mathrm{y}}\end{array}$ & $\begin{array}{l}1 \text { (Final) } \\
\mathrm{LS}_{2.4}-1.5 \mathrm{D}+\end{array}$ & \begin{tabular}{|l|}
0. \\
0
\end{tabular} & \begin{tabular}{|l|}
0. \\
0
\end{tabular} & $\begin{array}{l}15 \\
6 .\end{array}$ & \begin{tabular}{|l|}
0. \\
06
\end{tabular} & \begin{tabular}{|l|}
-18 \\
2.1
\end{tabular} & $\begin{array}{l}0 . \\
00\end{array}$ \\
\hline & & & & & $\begin{array}{l}\operatorname{Max} \\
F_{z}\end{array}$ & $\begin{array}{l}5951 \\
\text { (Final) } \\
\mathrm{LS}_{2.4-1.5 \mathrm{D}+}\end{array}$ & \begin{tabular}{l|}
0. \\
0 \\
0
\end{tabular} & \begin{tabular}{|l|}
0. \\
0 \\
0 \\
\end{tabular} & $\begin{array}{l}15 \\
6 . \\
55 \\
\end{array}$ & \begin{tabular}{|l|}
0. \\
06
\end{tabular} & \begin{tabular}{l|}
-18 \\
1.5 \\
6 \\
\end{tabular} & $\begin{array}{l}0 . \\
00\end{array}$ \\
\hline & & & & & $\begin{array}{l}\text { Min } \\
M_{x}\end{array}$ & $\begin{array}{l}106104 \\
\text { (Final) }\end{array}$ & \begin{tabular}{|l|}
0. \\
0
\end{tabular} & \begin{tabular}{|l|}
0. \\
0
\end{tabular} & $\begin{array}{l}86 \\
.1\end{array}$ & \begin{tabular}{|l|}
0. \\
02
\end{tabular} & \begin{tabular}{|l|}
-72 \\
.38 \\
\end{tabular} & $\begin{array}{l}0 . \\
00\end{array}$ \\
\hline & & & & & $\begin{array}{l}\text { Min } \\
F_{z}\end{array}$ & $\begin{array}{l}113104 \\
\text { (Final) }\end{array}$ & \begin{tabular}{|l|}
0. \\
0
\end{tabular} & \begin{tabular}{|l|}
0. \\
0
\end{tabular} & $\begin{array}{l}61 \\
.3\end{array}$ & \begin{tabular}{|l|}
0. \\
04
\end{tabular} & \begin{tabular}{|l|}
-47 \\
.29 \\
\end{tabular} & $\begin{array}{l}0 . \\
00\end{array}$ \\
\hline & & & & \multirow[t]{5}{*}{2} & $\begin{array}{l}\text { Max } \\
M_{y}\end{array}$ & $\begin{array}{l}40 \text { (Final) } \\
\mathrm{LS}_{2.3}-1.5 \mathrm{D}+\end{array}$ & \begin{tabular}{|l|}
0. \\
0
\end{tabular} & $\begin{array}{l}0 . \\
0\end{array}$ & $\begin{array}{l}15 \\
7 .\end{array}$ & $\begin{array}{l}-0 . \\
06\end{array}$ & \begin{tabular}{|l|}
18 \\
4.4
\end{tabular} & $\begin{array}{l}0 . \\
00\end{array}$ \\
\hline & & & & & $\begin{array}{l}\text { Min } \\
M_{x}\end{array}$ & $\begin{array}{l}4140 \\
\text { (Final) } \\
\text { LS }_{2.3}-1.5 \mathrm{D}+\end{array}$ & $\begin{array}{l}0 . \\
0 \\
0\end{array}$ & $\begin{array}{l}0 . \\
0 \\
0\end{array}$ & $\begin{array}{l}15 \\
6 . \\
24\end{array}$ & $\begin{array}{l}-0 . \\
08\end{array}$ & $\begin{array}{l}18 \\
2.2 \\
0\end{array}$ & $\begin{array}{l}0 . \\
00\end{array}$ \\
\hline & & & & & $\begin{array}{l}\operatorname{Max} \\
F_{z}\end{array}$ & $\begin{array}{l}5040 \\
\text { (Final) } \\
\text { LS }_{2.3}-1.5 \mathrm{D}+\end{array}$ & \begin{tabular}{|l|}
0. \\
0 \\
0
\end{tabular} & \begin{tabular}{|l|}
0. \\
0 \\
0
\end{tabular} & \begin{tabular}{|l|}
15 \\
7. \\
37
\end{tabular} & $\begin{array}{l}-0 . \\
06\end{array}$ & $\begin{array}{l}18 \\
3.8 \\
6\end{array}$ & $\begin{array}{l}0 . \\
00\end{array}$ \\
\hline & & & & & $\begin{array}{l}\text { Max } \\
M_{x}\end{array}$ & $\begin{array}{l}106104 \\
\text { (Final) }\end{array}$ & \begin{tabular}{|l|}
0. \\
0
\end{tabular} & $\begin{array}{l}0 . \\
0\end{array}$ & $\begin{array}{l}10 \\
3 .\end{array}$ & $\begin{array}{l}-0 . \\
02\end{array}$ & \begin{tabular}{|l|}
12 \\
0.9
\end{tabular} & $\begin{array}{l}0 . \\
00\end{array}$ \\
\hline & & & & & $\begin{array}{l}\text { Min } \\
F_{z}\end{array}$ & $\begin{array}{l}124115 \\
\text { (Final) }\end{array}$ & \begin{tabular}{|l|}
0. \\
0
\end{tabular} & $\begin{array}{l}0 . \\
0\end{array}$ & $\begin{array}{l}61 \\
.9\end{array}$ & $\begin{array}{l}-0 . \\
04\end{array}$ & $\begin{array}{l}48 . \\
22\end{array}$ & $\begin{array}{l}0 . \\
00\end{array}$ \\
\hline
\end{tabular}




\section{FINITE ELEMENT ANALYSIS:}

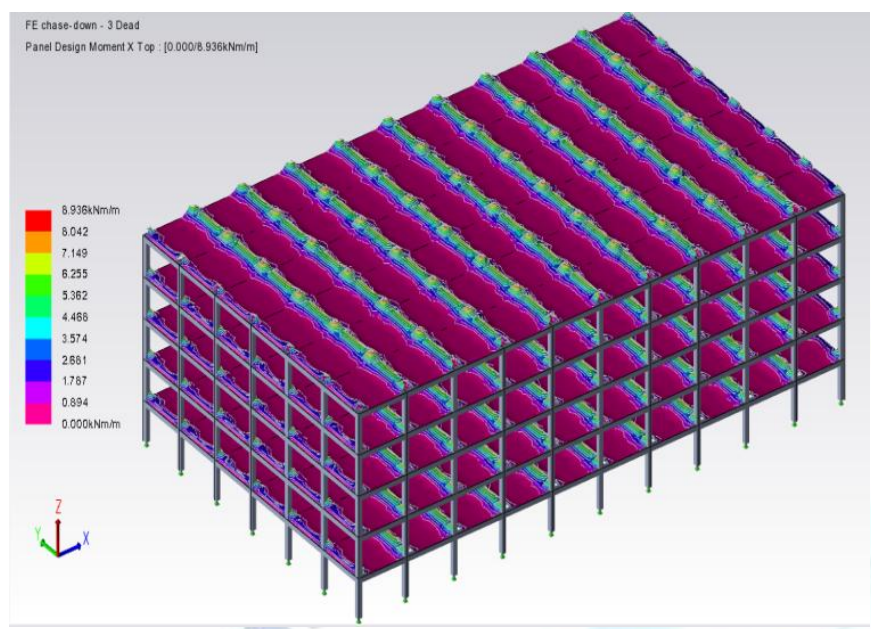

Panel Design Moment in a X-direction@ top

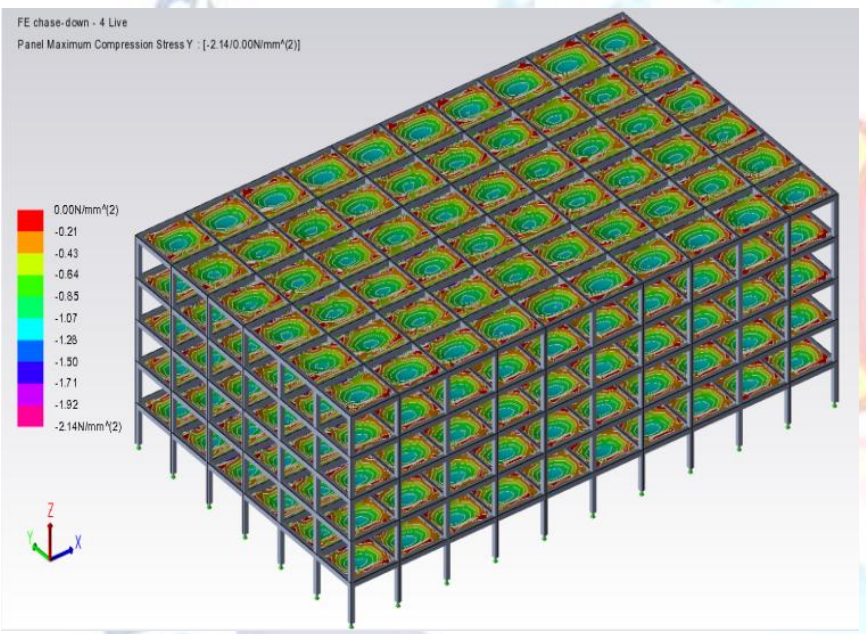

Panel Maximum Compression Stress in a Y-direction

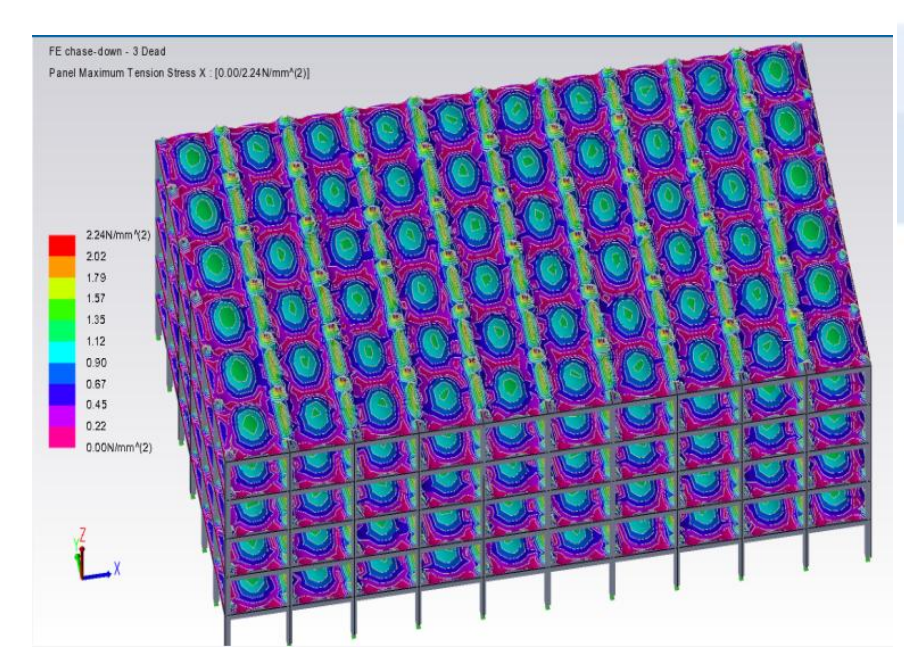

MaXimum Tension STREsS ON A PANEL IN X-DIRECTION

\section{Seismic Loading Summary:}

Structure details

Height to highest level

Dimension along Dir 1 at plinth level, dpis

Dimension along Dir 2 at plinth level, $d$ Dis Ignore seismic in floor (and below)

Number of storeys

Seismic Zone

Seismic Intensity

Zone factor, Z

Site Class

Percentage damping

Damping factor

Importance Factor, I

Effective Seismic Weight, W

Structure Type

Dirl

Dir2

Building System

Dir1

Dir2

Response Modification Factor, $R$

Approximate fundamental period, $T_{a}[\mathrm{sec}]$

Structure fundamental period, T [sec]

Average response spectrum coefficient, $S_{i} / g$

Design horizontal seismic coefficient, $A_{4}$

Base Shear (using $\left.T_{a}\right), V_{b}[k N]$

Base Shear (using T), $V_{B}[\mathrm{kN}]$

Modal Base Shear (from RSA), V: [kN]

Scaling Factor for Forces

\begin{tabular}{|c|c|c|}
\hline 16.000 & \multicolumn{2}{|l|}{$\mathrm{m}$} \\
\hline 60.000 & \multicolumn{2}{|l|}{$\mathrm{m}$} \\
\hline 36.000 & \multirow{2}{*}{\multicolumn{2}{|c|}{$\mathrm{m}$}} \\
\hline St. Base & & \\
\hline \multicolumn{3}{|l|}{ (Base) } \\
\hline II & & IS1893 (Part 1) \\
\hline Low & & Seismic Map \\
\hline 0.100 & & IS1893 (Part 1): \\
\hline \multicolumn{2}{|c|}{ Type II - Medium Soils } & 2016 Table 3 \\
\hline 5.00 & $\%$ & IS1893 (Part 1): \\
\hline 1.000 & & $\begin{array}{l}2016 \mathrm{Cl} .7 .2 .4 \\
\text { IS1893 (Part 1): }\end{array}$ \\
\hline 1.200 & & $\begin{array}{l}2016 \mathrm{Cl} .7 .2 .4 \\
\text { IS1893 (Part 1): }\end{array}$ \\
\hline 154039.2 & $\mathrm{kN}$ & $\begin{array}{l}2016 \text { Table \& } \\
\text { IS1893 (Part 1): }\end{array}$ \\
\hline 2 & & $\begin{array}{l}2016 \mathrm{Cl} .7 .6 .1 \\
\text { IS1893 (Part 1): }\end{array}$ \\
\hline \multicolumn{3}{|c|}{$\begin{array}{c}\text { RC MRF Buildings without any masonry infills } \\
\text { IS1893 (Part 1): }\end{array}$} \\
\hline \multirow{2}{*}{\multicolumn{3}{|c|}{$\begin{array}{l}\text { i) Moment Frame Systems } \\
\text { a) RC building with OMRF Table } 9 \\
\text { i) Moment Frame Systems } \\
\text { a) RC building with OMRF }\end{array}$}} \\
\hline & & \\
\hline Dirl & Dir2 & \\
\hline 3.000 & 3.000 & IS1893 (Part 1): \\
\hline 0.600 & 0.600 & $\begin{array}{l}2016 \text { Table 9 } \\
\text { IS1893 (Part 1): }\end{array}$ \\
\hline 1.476 & 1.505 & $2016 \mathrm{Cl} .7 .6 .2$ \\
\hline 0.922 & 0.903 & IS1893 (Part 1): \\
\hline 0.018 & 0.018 & $\begin{array}{l}2016 \text { Cl. } 6.42 \\
\text { IS1893 (Part 1): }\end{array}$ \\
\hline 6983.11 & 6983.11 & $\begin{array}{l}2016 \mathrm{Cl} .6 .4 .2 \\
\text { IS1893 (Part 1): }\end{array}$ \\
\hline 2839.38 & 2783.41 & $2016 \mathrm{Cl} .7 .6 .1$ \\
\hline 2637.27 & 2581.30 & \\
\hline 2.648 & 2.705 & IS1893 (Part 1): \\
\hline
\end{tabular}

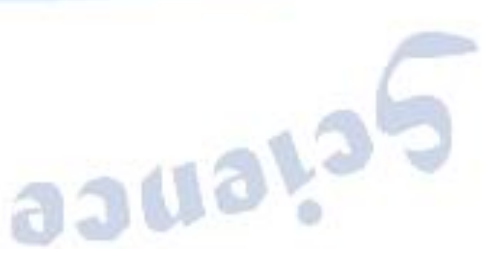




\begin{tabular}{|c|c|c|c|}
\hline Scaling Factor for Forces & 2.648 & 2.705 & IS1893 (Part 1): \\
\hline Scaling Factor for Drifts & 1.000 & 1.000 & $\begin{array}{l}2016 \mathrm{Cl} .7 .7 .3 \\
\text { IS1893 (Part 1): }\end{array}$ \\
\hline Structure Plan Irregularities - User Defined & & & $\begin{array}{l}2016 \mathrm{Cl} 1.7 .11 .1 .2 \\
\text { IS1893(Part 1): }\end{array}$ \\
\hline Plan irreg $\mathrm{i}$ - Torsional Irregularity & No & & 2016 Table 5 \\
\hline Plan irreg ii-Re-entrant comers & No & & \\
\hline Plan irreg iii-Floor slabs with excessive cut & No & & \\
\hline $\begin{array}{l}\text { outs/openings } \\
\text { Plan irreg iv - Out of Plane Offsets }\end{array}$ & No & & \\
\hline
\end{tabular}

\begin{tabular}{|c|c|c|}
\hline $\begin{array}{l}\text { Plan irreg v - Non parallel systems } \\
\text { Structure Vertical Irregularities - User Defined }\end{array}$ & No & IS1893 (Part1) \\
\hline 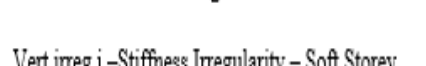 & $\mathrm{No}$ & 2016 Table 6 \\
\hline $\begin{array}{l}\text { Vert ineg 1-stumess integulanty - sort storey } \\
\text { Vert ineg ii -Mass Imegularity }\end{array}$ & $\begin{array}{l}\text { No } \\
\text { No }\end{array}$ & \\
\hline Vert irreg iii-Vertical Geometric Irregularity & No & \\
\hline Vert irreg iv -In-Plane Discontinuity in Vertical & No & \\
\hline $\begin{array}{l}\text { Elements Resisting Lateral Force } \\
\text { Vert ineg v-Strength Irregularity - Weak Storey }\end{array}$ & No & \\
\hline Vert irreg vi - Floating or Stub Columns & No & \\
\hline
\end{tabular}

Modal Response Spectrum Analysis is permitted (IS1893-Part 1 : 2016 Cl. 7.7.5)

\begin{tabular}{|l|r|r|c|r|r|r|r|r|}
\hline Refere & Lev & Weig & \multicolumn{4}{|r|}{ Dir1 } & \multicolumn{3}{|r|}{ Dir2 } \\
\cline { 4 - 9 } nce & el & $\mathbf{h t}$ & Fac & $\mathbf{Q}_{\mathbf{i}}$ & Ec & Fac & $\mathbf{Q}_{\mathbf{i}}$ & $\mathbf{E c}$ \\
& [m] & {$[\mathbf{k N ]}$} & tor & {$[\mathbf{k N}]$} & $\mathbf{c}$ & tor & {$[\mathbf{k N ]}$} & $\mathbf{c}$ \\
\hline St. 5 & 16. & 3028 & 1.1 & 313 & 1.8 & 1.1 & 313 & 3.0 \\
\hline St. 4 & 12. & 3100 & 0.7 & 205 & 1.8 & 0.7 & 205 & 3.0 \\
\hline St. 3 & 9.6 & 3091 & 0.4 & 115 & 1.8 & 0.4 & 115 & 3.0 \\
\hline St. 2 & 6.4 & 3091 & 0.1 & 512. & 1.8 & 0.1 & 512. & 3.0 \\
\hline St. 1 & 3.2 & 3091 & 0.0 & 128. & 1.8 & 0.0 & 128. & 3.0 \\
\hline
\end{tabular}

\section{TORSION}

\section{General:}

Forces that cause a member to twist about a longitudinal axis are called torsional loads. Simple torsion is produced only by a couple, or moment, in a plane perpendicular to the axis. If a couple lies in a non-perpendicular plane, it can be resolved into a torsional moment, in a plane perpendicular to the axis, and bending moments, in planes through the axis.

\section{Shear Diagrams:}

The unbalanced external vertical force at a section is called the shear. It is equal to the algebraic sum of the forces that lie on either side of the section. Upward acting forces on the left of the section are considered positive, downward forces negative; signs are reversed for forces on the right.

A diagram in which the shear at every point along the length of a beam is plotted as an ordinate is called a shear diagram.

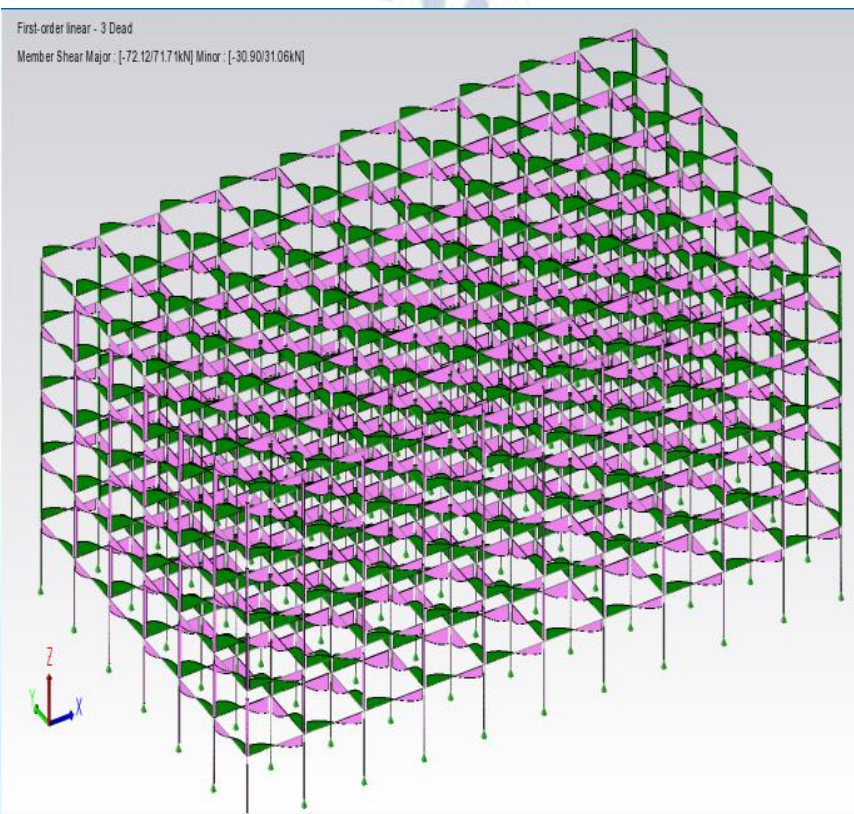

Shear Diagram for the Beams with Loads

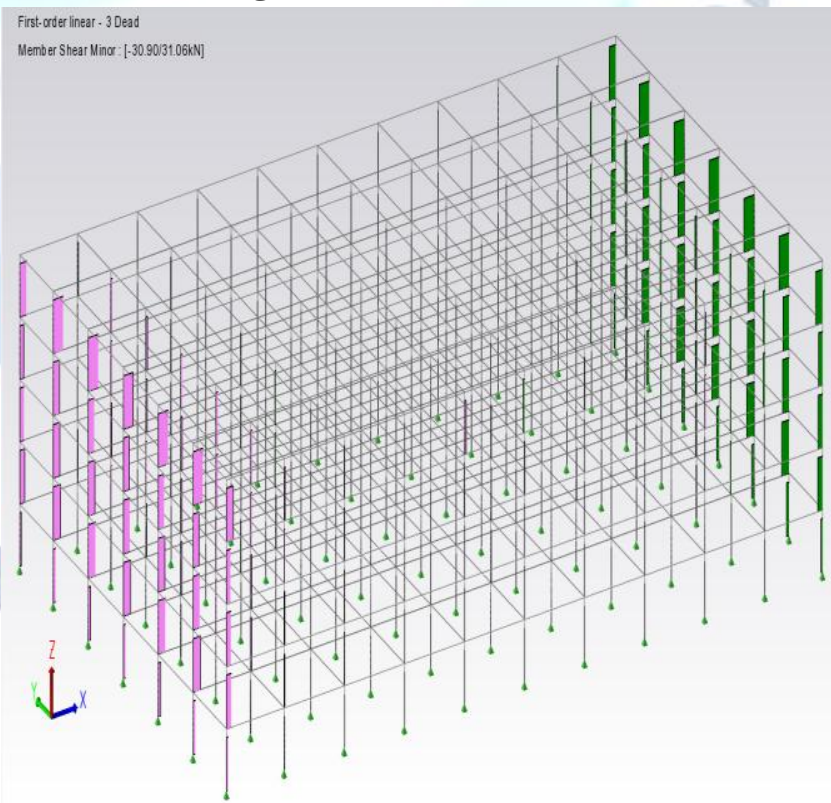

Member Shear Minor 


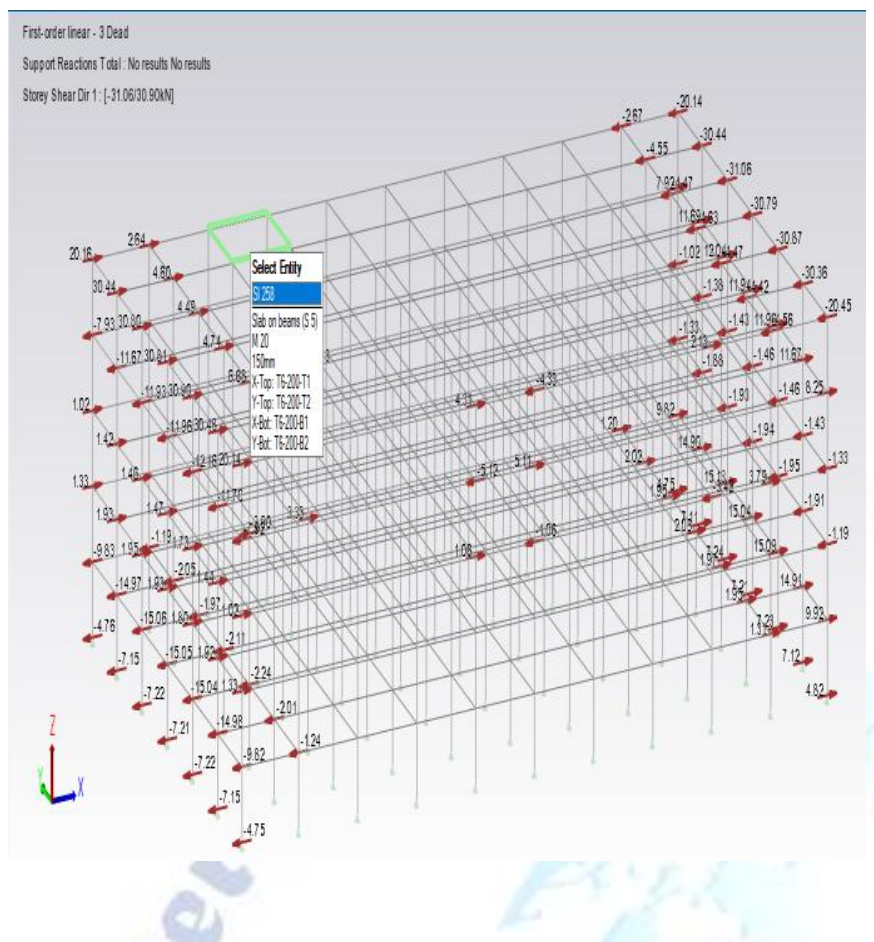

Shear stress will be formed in Direction-1

\section{Bending-Moment Diagrams:}

The unbalanced moment of the external forces about a vertical section through a beam is called the bending moment. It is equal to the algebraic sum of the moments about the section of the external forces that lie on one side of the section. Clockwise moments are considered positive, counterclockwise moments negative, when the forces considered lie on the left of the section. Thus, when the bending moment is positive, the bottom of the beam is in tension. A diagram in which the bending moment at every point along the length of a beam is plotted as an ordinate is called a bending-moment diagram. The bending moment at the supports for this simply supported beam obviously is zero. Between the supports and the first load, the bending moment is proportional to the distance from the support, since it is equal to the reaction times the distance from the support. Hence the bending-moment diagram for this portion of the beam is a sloping straight line.

If the bending moment and shear are known at any section of a beam, the bending moment at any other section may be computed, providing there are no unknown forces between the two sections. The rule is: The bending moment at any section of a beam is equal to the bending moment at any section to the left, plus the shear at that section times the distance between sections, minus the moments of intervening loads. If the section with known moment and share is on the right, the sign convention must be reversed.

\section{v. CONCLUSION}

The design of column, beam, footing and slabs are done in limit state method which is safe at control of deflection and in all aspects using Tekla software, the design considerations has been taken as per IS Codes. The design is safe in all conditions on comparison with drawing, manual design and the geometrical model using Tekla. The area of Ast required for the beam, column, footing and slab are comparatively similar to that of requirement.

While software also gives required sizes of bar and members but with factor of safety.

Tekla has the capability to calculate the reinforcement needed for any concrete section. The program contains a number of parameters which are designed asper IS456:2000. Beams are designed for flexure, shear $\&$ torsion.

From the Tekla result required different types size and number of bars are found. And final evaluation and valuation is confirmed by estimation and costing software.

Due to manual and software calculations, the required amount and material will be minimized. So the structure comes under healthy for environment and safe for human beings.

It concludes that the efficiency and reliability of the software in the field of designing is much better to that of the manual work. It has been seen that the software generated results were more efficient and economical which included the various different conditions under the designing conditions which are difficult to consider when done manually.

Tekla structural designer includes full-fledged documentation and reporting capabilities, and these, along with its ability to work with both concrete and steel structures, extensive analysis and design capabilities, a Finite Element engine that enables it to perform complex and accurate analyses, the ease of creating loads and combining them into load cases and combinations, the automated design capabilities, and the ability to review analysis results in detail to fix errors and over design all add up to a structural analysis and design application that is top of its class.

The theory of LSM which provide adequate strength, serviceability, and durability besides economy. The displacement, shear force, bending moment variation has been shown. If any beam fails, the dimensions of beam and column should 
be changed and reinforcement detailing can be produced.

\section{REFERENCES}

[1] Massumi A and Mohammadi R, "Structural redundancy of 3D RC frames under seismic excitations", Structure Engineering And Mechanics, Vol.9, No.1, pp.15-36, 2016.

[2] Gorgun H, "The stability of semi-rigid skeletal structures accounting for shear Deformation", Structure Engineering And Mechanics, Vol.57, No.6,pp.1065-1084, 2016.

[3] Parulekar Y.M, Reddy G.R, Singh R.K, Gopalkrishnan N and Ramarao G.V, "Seismic performance evaluation of mid-rise shear walls: experimental and analysis", Structure Engineering and Mechanics, Vol. 59, pp.291-312, 2016.

[4] Standard I. Plain and Reinforced Concrete-Code of Practice (IS-456:2000).Bureau of Indian Standards, New Delhi, July2000.

[5] Bureau of Indian Standards(IS1893), Part-1: Criteria for Earthquake Resistant Design of Structures: General Provisions and Buildings.

[6] Wilson E.L. and Habibulah A., "SAP2000 integrated finite element analysis and design of structures, Analysis reference, Computers and Structures, 1997.

[7] Fire Safety of Buildings (General): Details of Construction IS:16421989, Bureau of Indian Standards, New Delhi, 1989.

[8] Bureau of Indian Standards(IS1893), Part-1: Criteria for Earthquake Resistant Design of Structures: General Provisions and Buildings.

[9] www.Google.com

[10] Google Wikipedia.

[11] Reinforced Concrete Designer's Handbook, 10 th Edition, by C.E.Reynolds and J.C.Steedman, E \& FN SPON, London, 1997.

[12] Indian Standard Plain and Reinforced Concrete - Code of Practice (4th Revision), IS 456: 2000, BIS, New Delhi.

[13] Design Aids for Reinforced Concrete to IS: 456 - 1978, BIS, New Delhi.

[14] Reinforced Concrete Limit State Design, $6^{\text {th }}$ Edition, by Ashok K. Jain, Nem Chand \& Bros, Rourke, 2002.

[15] Limit State Design of Reinforced Concrete, $2^{\text {nd }}$ Edition, by P.C.Varghese, Prentice-Hall of India Pvt. Ltd., New Delhi, 2002.

[16] American Concrete Institute - Building Code Requirements for Structural Concrete (ACI 318-05) and Commentary (ACI 318R-05).

[17] American Concrete Institute - Building Code Requirements for Structural Concrete (ACI 318-08) incorporating ERRATA dated July 26th 2010.

[18] American Concrete Institute - Building Code Requirements for Structural Concrete (ACI 318-11).

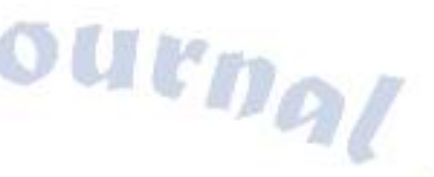

